\title{
Comportamento de Cura de Adesivo Epoxídico Contendo Grupo Mercaptana Avaliado por Espectroscopia no Infravermelho (MIR/NIR) e Calorimetria Exploratória Diferencial (DSC)
}

\author{
Hilzette P. C. Andrade, Milton F. Diniz, Margarete F. P. Azevedo, Silvana N. Cassu \\ Instituto de Aeronáutica e Espaço, CTA \\ Vera L. Lourenço, Rita C. L. Dutra \\ Instituto de Aeronáutica e Espaço, CTA \\ Instituto Tecnológico da Aeronáutica - ITA, CTA
}

\begin{abstract}
Resumo: No presente trabalho, a flexibilidade de um adesivo epoxídico contendo diglicidiléter de bisfenol A (DGEBA) e dietilenotriamina (DETA) como agente de cura foi modificada pela adição de um segundo componente contendo grupos mercaptana (CAPCURE). A adição de amianto ao adesivo contendo CAPCURE também foi avaliada. As reações entre os grupos epoxídicos e os grupos amina, assim como entre os grupos epoxídicos e os grupos mercaptana, foram estudadas nas regiões espectrais do infravermelho médio (MIR) e próximo (NIR). Observou-se que o amianto não interfere nas reações de cura e que a espectroscopia FT-NIR evidencia melhor as alterações espectrométricas ocorridas durante as reações em relação à análise FT-MIR. O tempo das reações de cura foi monitorado por calorimetria exploratória diferencial (DSC), observandose que a introdução do CAPCURE acelerou a cura da resina. A energia de ativação $\left(\mathrm{E}_{\mathrm{a}}\right)$ das reações de cura foi obtida pelos métodos de Barrett e Borchardt-Daniels. Os adesivos contendo CAPCURE mostraram $\mathrm{E}_{\mathrm{a}}$ em torno de $30 \mathrm{~kJ}^{\mathrm{mol}}{ }^{-1}$, enquanto $o$ adesivo DGEBA/DETA apresentou $\mathrm{E}_{\mathrm{a}}$ de $46 \mathrm{~kJ} \mathrm{~mol}^{-1}$, ambas calculadas pelo método de Barrett.
\end{abstract}

Palavras-chave: Adesivo, resina epoxídica, mercaptana, MIR, NIR, DSC.

Cure Behavior of Epoxy Adhesive Containig Mercaptan Group Evaluated by Infrared Spectroscopy (MIR/NIR) and Differential Scanning Calorimetry (DSC)

Abstracts: In the present work, the flexibility of an epoxy adhesive containing diglycidylether of bisphenol-A (DGEBA) and diethylenetriamine (DETA) as curing agent was changed by the addition of a second component containing mercap$\tan$ groups (CAPCURE). The addition of asbestos as a filler in the adhesive containing CAPCURE was also evaluated. Epoxy-amine and epoxy-mercaptan reactions were studied in NIR and MIR spectral regions. The filler addition did not cause influence on the cure reactions and spectrometric changes of cure reactions could be better observed by FT-NIR than FT-MIR analysis. The cure reaction time was monitored by DSC experiments and it was observed that the introduction of CAPCURE accelerated the cure reaction. The activation energies $\left(\mathrm{E}_{\mathrm{a}}\right)$ of curing reactions were obtained using Barrett and Borchardt-Daniels methods. The adhesives containing CAPCURE showed $\mathrm{E}_{\mathrm{a}}$ around $30 \mathrm{~kJ} \cdot \mathrm{mol}^{-1}$, while DGEBA/DETA adhesive presented $\mathrm{E}_{\mathrm{a}}$ of $46 \mathrm{~kJ}^{\mathrm{mol}}{ }^{-1}$ calculated by Barrett method.

Keywords: Adhesive, epoxy, mecaptan, MIR, NIR, DSC.

\section{Introdução}

Os lançadores de satélite e sondas desenvolvidos atualmente no instituto de aeronáutica e espaço (IAE), utilizam propelente sólido, o que causa um aquecimento excessivo das paredes metálicas do motor-foguete. Para minimizar este efeito, as partes mais críticas do motor-foguete são protegidas do intenso calor do ambiente por proteções térmicas rígidas, que são compósitos à base de resina fenólica e tecidos de carbono ou amianto. Essas proteções variam em tamanho, espessura e configuração, dependendo da sua localização e algumas delas são coladas à estrutura metálica com adesivos à base de resina epoxídica. Adesivos epoxídicos são utilizados em interfaces tais como, metal/metal, carbono-carbono/alumínio e grafite/alumínio. Como a interface adesiva é exposta a altas solicitações mecânicas durante os lançamentos há a necessidade de acomodação das tensões geradas, evitando-se o aparecimento de trincas frágeis e catastróficas na região de colagem. Os compostos mais conhecidos, que promovem as reações de cura de resinas epoxídicas, são as aminas, amidas e anidridos, entretanto, mercaptanas, como o polissulfeto, têm sido utilizados neste processo ${ }^{[1-3]}$. Durante as últimas décadas, o polissulfeto foi adicionado ao adesivo epoxídico para aumentar sua flexibilidade. Quando o polissulfeto líqui-

Autor para correspondência: Rita C. L. Dutra, Divisão de Química, Comando-Geral de Tecnologia Aeroespacial, Praça Mal. Eduardo Gomes 50, 
do é adicionado à resina epoxídica, seus grupos mercaptana terminais reagem com os grupos oxirano da resina epoxídica, e são incorporados à matriz conferindo flexibilidade ao adesi$\mathrm{vo}^{[1,4]}$. Entretanto, este produto não é produzido nacionalmente, sendo de difícil aquisição. Um substituto promissor ao polissulfeto é um polímero liquido comercial contendo grupos mercaptana, CAPCURE $3-800^{\circledR}$, que foi utilizado anteriormente como agente de cura em sistemas epoxídicos ${ }^{[5-7]}$.

O monitoramento dos grupos formados na reação de cura é essencial para estabelecer a relação estrutura-propriedade e otimizar as condições de utilização dos materiais finais ${ }^{[8]}$. A espectroscopia no infravermelho (IR), nas regiões MIR e NIR tem sido utilizada ${ }^{[8-12]}$ para avaliação de reações de cura em resinas epoxídicas. Características, vantagens e desvantagens dessas técnicas são discutidas na literatura ${ }^{[7,9,10]}$ para sistemas epoxídicos. A banda MIR sugerida para o acompanhamento de reação de cura é a relativa ao grupo epóxido terminal, em $917 \mathrm{~cm}^{-1}$, enquanto, na região NIR, bandas nas regiões de 4545 e $8628 \mathrm{~cm}^{-1}$ são atribuídas a esse grupo. Segundo Weyer $^{[13]}$, o acompanhamento da reação de cura em resina epoxídica por meio da banda em $4545 \mathrm{~cm}^{-1}$ na região NIR, mostra menor interferência do que a vibração de deformação do anel epóxido na região MIR, além da possibilidade da reação poder ser acompanhada com teor mais baixo de grupos epóxido.

Outra técnica usualmente utilizada para acompanhamento de cura, é a calorimetria diferencial exploratória (DSC). O comportamento térmico de sistemas epoxídicos tem sido descrito na literatura em relação à influência de cargas nos parâmetros de cura ${ }^{[14]}$, influência de agentes de reticulação e adição de plastificantes ${ }^{[15]}$, e efeitos de condições de cura $^{[16]}$.

Deste modo, com base nas características das citadas técnicas instrumentais, neste trabalho, avaliou-se a influência do CAPCURE- $3800^{\circledR}$ na cura da diglicidiléter de bisfenol A (DGEBA) na presença do agente de cura dietilenotriamina (DETA), por meio de espectroscopia FT-MIR e FT-NIR e por DSC. O efeito da adição de amianto nas condições de cura também foi avaliado. Na metodologia proposta, as análises FT-MIR/NIR foram utilizadas qualitativamente para a verificação de alterações espectrométricas ocorridas durante a reação. A energia de ativação para a reação de cura dos adesivos foi calculada a partir das curvas DSC utilizando os métodos de Borchardt e Daniels ${ }^{[16]}$ e de Barrett ${ }^{[17]}$.

\section{Experimental}

\section{Material}

Os materiais utilizados na obtenção dos adesivos, bem como seus códigos, fabricantes e propriedades são apresentados na Tabela 1. As formulações utilizadas em cada adesivo são mostradas na Tabela 2.

Os adesivos foram preparados misturando-se a resina DGEBA, o CAPCURE e o amianto, no caso do adesivo contendo carga. $\mathrm{O}$ agente de cura, DETA, foi o último componente adicionado à mistura. $\mathrm{O}$ adesivo foi colocado em um
Tabela 1. Materiais utilizados para a obtenção dos adesivos.

\begin{tabular}{ccccc}
\hline Material & Sigla & Código & Fornecedor & $\begin{array}{c}\text { EEW } \\
\left(\text { g.eq }^{-1}\right)\end{array}$ \\
\hline $\begin{array}{c}\text { Diglicidiléter } \\
\text { de bisfenol A }\end{array}$ & DGEBA & XGY-1109 & Maxiepoxi & $\begin{array}{c}200- \\
213\end{array}$ \\
$\begin{array}{c}\text { Dietileno } \\
\text { triamina }\end{array}$ & DETA & HY-950 & Maxiepoxi & - \\
CAPCURE & CAP & $\begin{array}{c}\text { CAPCURE } \\
3-800^{\circledR}\end{array}$ & Cognis & 333 \\
Amianto & AM & OB 80303 & Minérios & - \\
& & & Ouro & \\
& & & Branco & \\
\hline
\end{tabular}

Tabela 2. Composição dos adesivos epoxídicos.

\begin{tabular}{ccccc}
\hline Adesivo & $\begin{array}{c}\text { DGEBA } \\
(\mathbf{p h r})\end{array}$ & $\begin{array}{c}\text { DETA } \\
(\mathbf{p h r})\end{array}$ & $\begin{array}{c}\text { AM } \\
(\mathbf{p h r})\end{array}$ & $\begin{array}{c}\text { CAP } \\
(\mathbf{p h r})\end{array}$ \\
\hline DGEBA/DETA & 100 & 10 & - & - \\
CAP & 50 & 10 & - & 50 \\
CAP/AM & 50 & 10 & 50 & 50 \\
\hline
\end{tabular}

*phr $=$ partes por 100 partes de DGEBA.

molde de aço inox de $25 \mathrm{~cm}$ de diâmetro revestido por um filme fino de TEFLON, mantido a $25{ }^{\circ} \mathrm{C}$ durante a cura do adesivo.

\section{Análise por FT-IR das misturas reacionais e seus materiais de partida}

Para a obtenção dos espectros na região de infravermelho foi utilizado o FT-IR Spectrum One PERKINELMER com as seguintes condições: região espectral 4000 a $400 \mathrm{~cm}^{-1}$ (MIR) e 6500 a $4000 \mathrm{~cm}^{-1}$ (região espectral parcial NIR), resolução $4 \mathrm{~cm}^{-1}$, ganho 1 e 40 varreduras. As análises foram feitas a partir do tempo inicial de mistura, considerado tempo zero, e após 2 horas até 6 horas de reação. Após esse período os ensaios foram realizados em intervalos de 24 horas. Foram feitas duplicatas das análises.

Amostras líquidas, puras e como mistura reacional, dos materiais de partida foram analisadas nas regiões MIR e NIR como filmes líquidos, a $25{ }^{\circ} \mathrm{C}$ nos intervalos de tempo especificados. Amostras sólidas de materiais de partida foram analisadas como pastilhas de $\mathrm{KBr}(1: 400 \mathrm{mg})$. Para a análise NIR, as amostras foram preparadas em células de CsI.

\section{Análise por DSC das misturas reacionais e seus materiais de partida}

As análises calorimétricas foram realizadas em um DSC-Q 100 da TA Instruments. Os materiais puros, bem como os adesivos, foram aquecidos entre -90 e $250{ }^{\circ} \mathrm{C}$ a $10{ }^{\circ} \mathrm{C} / \mathrm{min}$, sob atmosfera de nitrogênio. $\mathrm{O}$ intervalo de tempo entre as análises foi o mesmo descrito para as análises FT-IR. Os valores da temperatura de transição vítrea (Tg) dos adesivos foram obtidos no segundo aquecimento. 


\section{Resultados e Discussão}

\section{Análises FT-MIR/NIR dos materiais de partida e dos adesivos}

Neste trabalho acompanhou-se por espectroscopia FT-MIR/NIR a evolução das reações de cura entre os grupos epoxídicos, que podem reagir tanto com os grupos amina, presentes no agente de cura DETA, como com os grupos mercaptana, presentes no CAPCURE (Figura 1).

Considerando-se que as bandas da resina epoxídica tipo DGEBA são extensivamente discutidas na literatura ${ }^{[9]}$, e que as absorções características do CAPCURE, já foram atribuídas anteriormente ${ }^{[7]}$, neste trabalho somente serão avaliadas as alterações espectrométricas MIR e NIR do DETA e do amianto. A Figura 2a mostra os espectros MIR dos materiais DEGBA, CAPCURE, DETA e amianto OB 8030. As bandas observadas no espectro MIR do DETA ocorrem em: $3422 \mathrm{~cm}^{-1}$ ( $v$ NH e/ou OH umidade), $1656 \mathrm{~cm}^{-1}\left(\delta \mathrm{NH}_{2}\right.$ e/ou $\delta \mathrm{OH}), 1602 \mathrm{~cm}^{-1}\left(\delta \mathrm{NH}_{2}\right)$ e $952 \mathrm{~cm}^{-1}\left(\omega \mathrm{NH}_{2}\right)$. Estas bandas são características de amina com estrutura de dietilenotrietilenoamina, de acordo com a literatura ${ }^{[19 a]}$. As principais bandas de amianto estão em: $3675 \mathrm{~cm}^{-1}(\mathrm{vOH})$ e 1068-951 $\mathrm{cm}^{-1}(\mathrm{~V} \mathrm{Si-O})^{[19 b]}$.

As bandas NIR da resina epoxídica já foram atribuídas aos seus modos vibracionais de acordo com Poisson ${ }^{[12]}$ e descritas em trabalho anterior ${ }^{[20]}$. A banda em $4530 \mathrm{~cm}^{-1}$, atribuída ao grupo oxirano foi utilizada para acompanhar a reação de cura nos adesivos. De forma similar, as bandas NIR observadas para o CAPCURE já foram discutidas em trabalho anterior ${ }^{[20]}$. As absorções acima de $5000 \mathrm{~cm}^{-1}$ podem ser atribuídas ao primeiro sobretom da banda em $2654 \mathrm{~cm}^{-1}$, portanto, a banda em $5209 \mathrm{~cm}^{-1}$, foi utilizada como característica do CAPCURE para acompanhar a reação entre os grupos epoxídicos da resina e o grupo mercaptana. Com relação à análise NIR de aminas similares à utilizada na mistura reacional, é citado ${ }^{[21]}$ que a banda de combinação de estiramento e deformação $\mathrm{NH}$ ocorre próxima a $5000 \mathrm{~cm}^{-1}$. No espectro
NIR do DETA é observada uma banda em $4934 \mathrm{~cm}^{-1}$, que foi, então, utilizada para o acompanhamento da reação de cura entre os grupos oxirano da resina epoxídica e os grupos da amina (Figura 2b). As principais alterações espectrométricas FT-NIR observadas para o sistema CAP nos diferentes tempos são mostradas na Figura 2c, e sugerem algum consumo dos grupos oxirano da resina e $\mathrm{NH}$ da amina, antes de 24 horas de reação.

A Figura 3a, que mostra os espectros MIR da mistura reacional do adesivo CAP no tempo inicial de cura e após intervalos determinados de tempos de reação, mostra que as mesmas alterações espectrométricas MIR foram observadas, independentemente, do uso da carga amianto no sistema.

Os espectros NIR da mistura reacional do adesivo CAP/AM inicial e após intervalos determinados de tempos de reação, assim como os espectros MIR mostram que, as mesmas alterações espectrométricas foram observadas, independentemente do uso da carga no sistema (Figura 3b).

Pela comparação entre os resultados obtidos pelos espectros nas regiões NIR e MIR, observou-se que as alterações espectrométricas encontradas na região NIR estão em maior número (bandas para resina e amina) e são mais nítidas, permitindo melhor estimativa do tempo de cura que as assinaladas na região MIR.

\section{Análise calorimétrica}

As análises DSC foram realizadas com a finalidade de se determinar o tempo total da reação de cura ou reticulação do adesivo, simulando-se uma camada aplicada similar à utilizada nas colagens em motor-foguete. Essa simulação é importante, pois como a reação do sistema epoxídico é autocatalítica ${ }^{[22]}$, depende da relação massa/volume da amostra. Estas análises buscaram determinar o tempo mínimo necessário para que o material possa ser submetido a solicitações mecânicas e térmicas com máximo desempenho, ou seja, após sua cura completa ou a estabilização da reação de cura a $25{ }^{\circ} \mathrm{C}$.

D GEB A
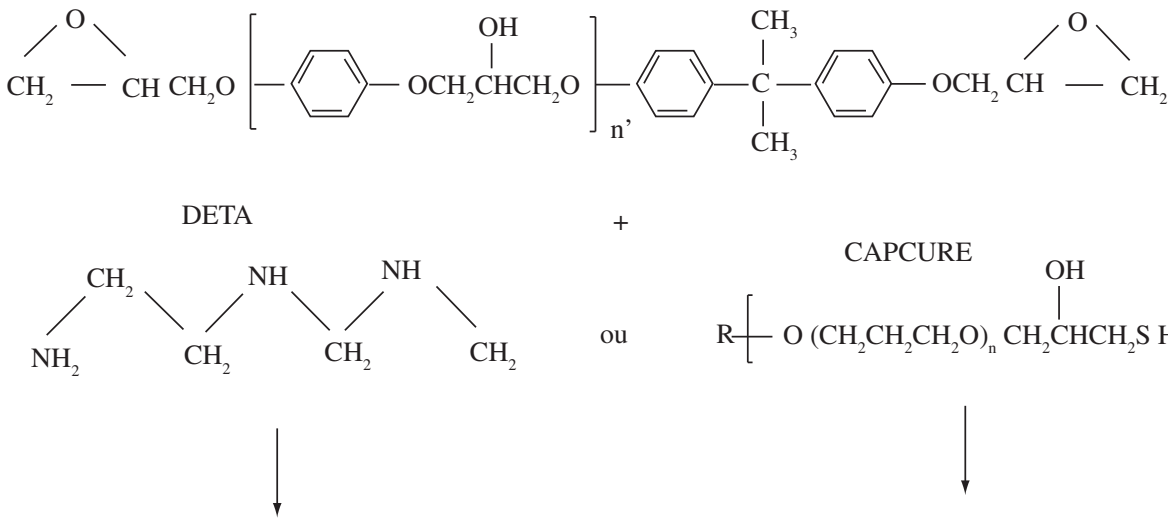

$+$

ou
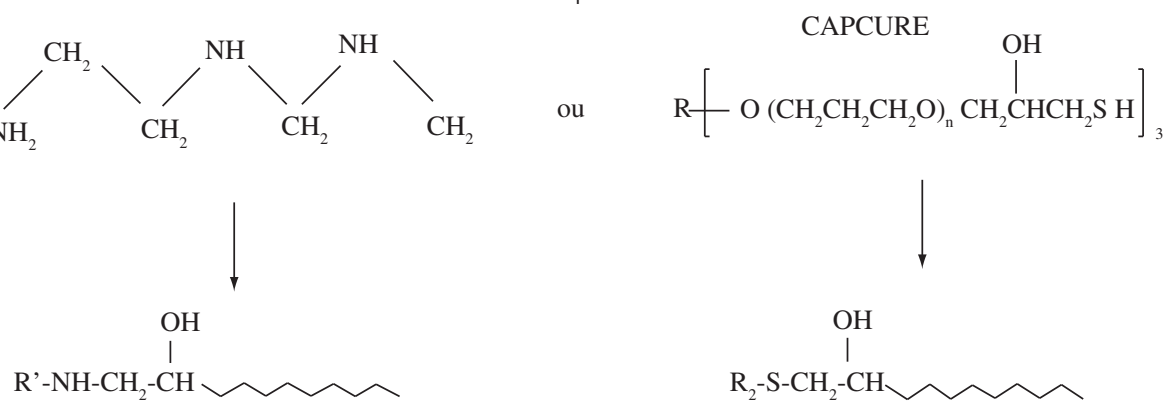

Figura 1. Reações envolvendo a resina DGEBA durante a cura dos adesivos CAP e CAP/AM ${ }^{[2,18]}$. 


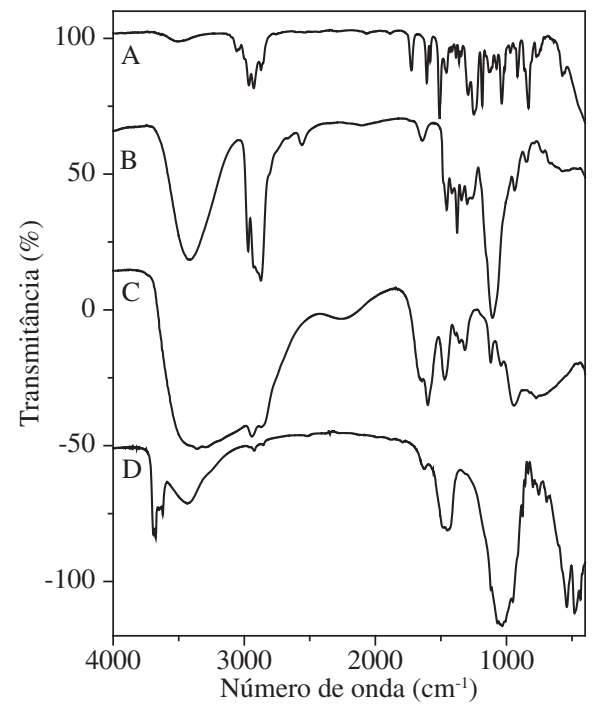

(a)

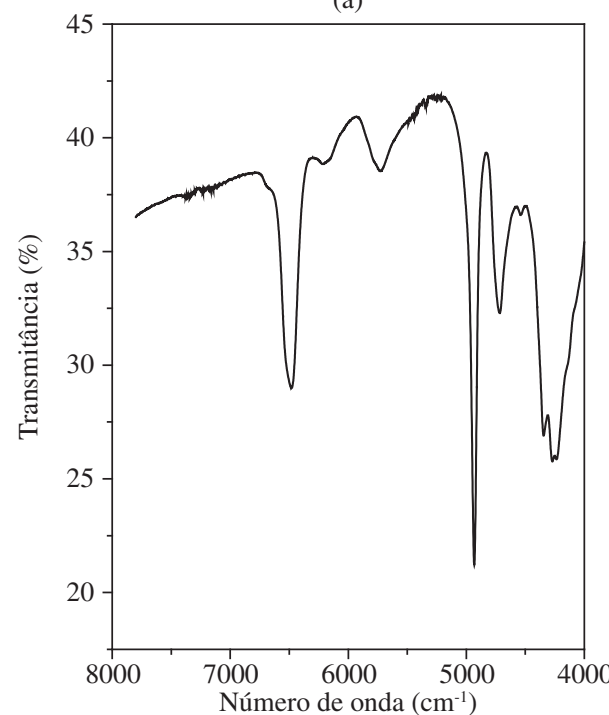

(b)

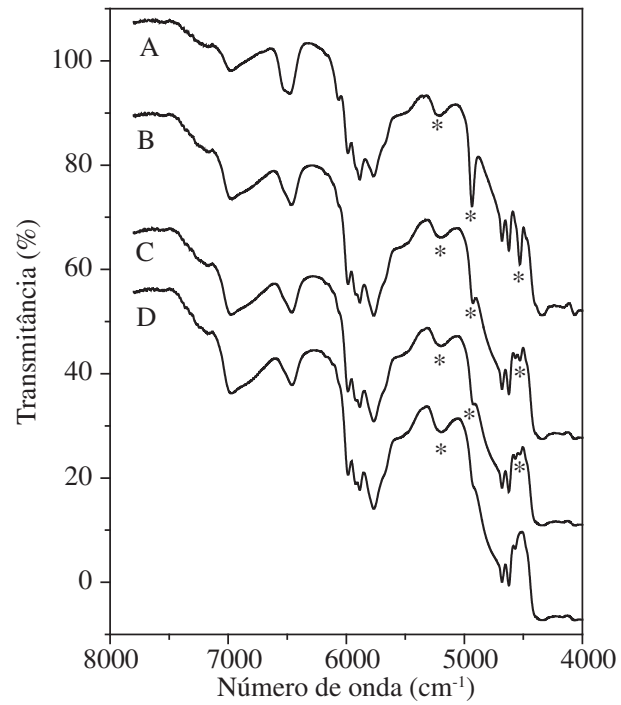

(c)

Figura 2. a) Espectros FT-MIR: (A) DGEBA, (B) CAPCURE, (C) DETA e (D) amianto; b) espectros FT-NIR de DETA; e c) espectros FT-NIR do adesivo CAP nos tempos (horas): (A) 0, (B) 4, (C) 6 e (D) 24.

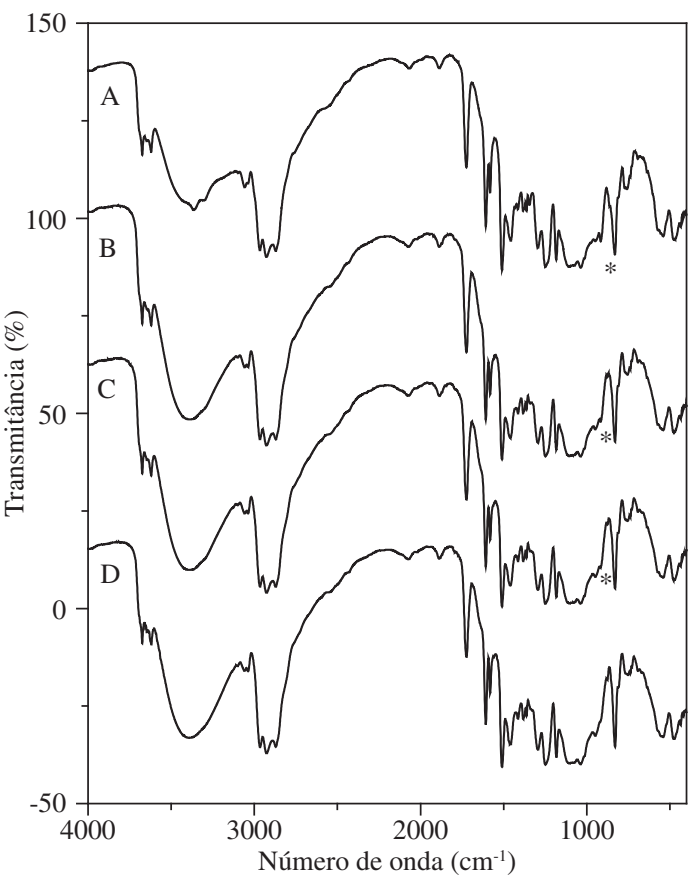

(a)

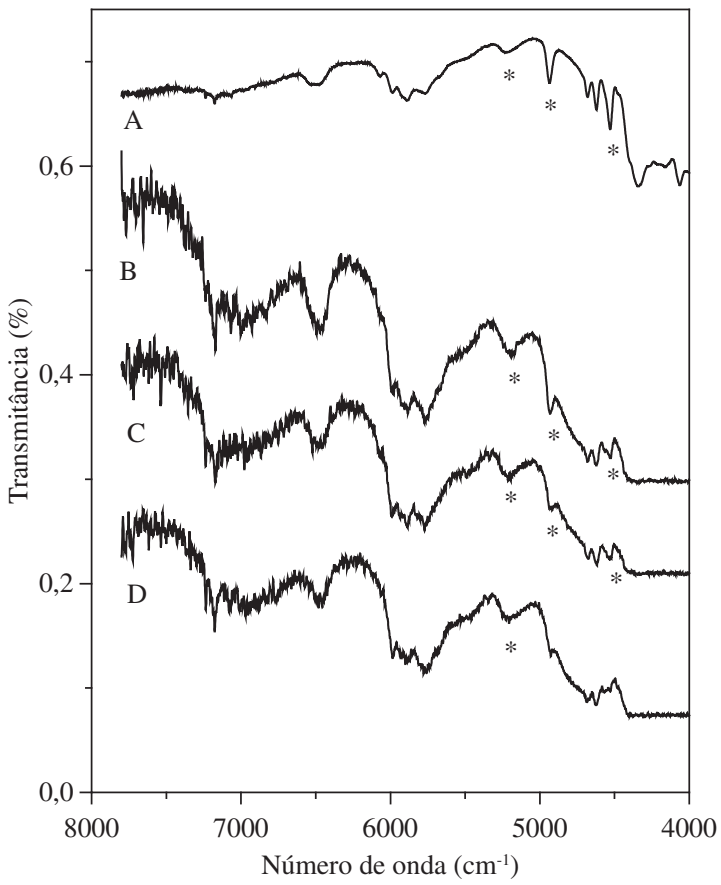

(b)

Figura 3. a) Espectros FT-MIR da mistura reacional do adesivo CAP/AM nos tempos (horas): (A) 0, (B) 4, (C) 6 e (D) 24; e b) espectros FT-NIR da mistura reacional do adesivo CAP/AM nos tempos (horas):(A) 0, (B) 4, (C) 6 e (D) 24.

As análises DSC foram realizadas para o adesivo DGEBA/DETA que foi utilizado como referência e para os adesivos CAP e CAP/AM. As curvas DSC obtidas durante o primeiro aquecimento apresentam um pico exotérmico que passa por um máximo variando entre 105 e $122{ }^{\circ} \mathrm{C}$ para o sistema DGEBA/DETA e 95 e $108{ }^{\circ} \mathrm{C}$ para os adesivos CAP e 
CAP/AM, e apresenta uma redução na intensidade com o aumento do tempo de reação (Figura 4). Este comportamento é esperado, uma vez que, a cura da resina avança com o tempo causando uma redução da entalpia que está sendo medida em relação ao material não reagido. Observa-se pela Figura 4b que após 24 horas o adesivo CAP está completamente curado, não se observando mais o pico exotérmico referente à cura residual. A adição do amianto ao adesivo contendo o CAPCURE praticamente não alterou o perfil das curvas DSC, por esta razão estas curvas não foram apresentadas. No caso do adesivo DGEBA/DETA (Figura 4a) nota-se que há uma redução da cura residual até 24 horas, indicando que nesse intervalo de tempo a reação prossegue a temperatura ambiente. Após esse período a intensidade do pico exotérmico referente à cura residual se mantém constante até 96 horas, sugerindo que no sistema DGEBA/DETA a reação de cura se torna muito lenta à temperatura ambiente.

$\mathrm{Na}$ Figura 4 pode-se observar que antes do pico exotérmico de cura, há a região de transição vítrea, notando-se que ocorre um aumento na temperatura de transição vítrea $(\mathrm{Tg})$ com o aumento do grau de reticulação (maiores tempos de reação), o que pode ser melhor visualizado na Figura 4c. No caso do adesivo DGEBA/DETA a um determinado grau de cura a Tg ultrapassa a temperatura ambiente e continua a subir até que a reação de cura se estabiliza. Como consequiência, a mobilidade das cadeias que ainda não reagiram é reduzida a temperatura ambiente, diminuindo a velocidade da reação de cura que tende a se estabilizar se a temperatura permanecer constante. No caso dos adesivos CAP e CAP/AM a temperatura de transição vítrea não ultrapassa a temperatura ambiente (Figura 4c). Esta redução na temperatura de transição vítrea deve ser atribuída à incorporação das cadeias flexíveis do CAPCURE no adesivo DGEBA/DETA.

Como a finalidade deste trabalho era monitorar o tempo de cura do adesivo nas condições de aplicação $\left(\right.$ a $25{ }^{\circ} \mathrm{C}$ ), os experimentos DSC foram realizados a $10^{\circ} \mathrm{C} / \mathrm{min}$ para cada composição nos intervalos definidos de tempo. Dessa forma, foram aplicados os tratamentos cinéticos que utilizam apenas um experimento DSC obtido a taxa de aquecimento constante, como os métodos de Barrett ${ }^{[17]}$ e de Borchardt e Daniels ${ }^{[16]}$. Esses métodos são bem semelhantes, enquanto o tratamento de Borchardt e Daniels é aplicável a reações com ordem $\mathrm{n} \leq 2$, o método de Barrett assume cinética de primeira ordem ${ }^{[16,17]}$. Nenhum dos dois é totalmente adequado para obtenção de valores reais de energia de ativação ou ordem de reação de sistemas autocatalíticos, como é o caso de sistemas epoxídicos, no entanto, podem ser utilizados na avaliação dos perfis de comportamento.

O método cinético de Barrett foi aplicado às curvas DSC dos adesivos epoxídicos, obtidas no início da reação de cura. Este método foi utilizado por Miranda e colaboradores ${ }^{[23]}$ para avaliar o efeito da adição de diferentes teores de sílica na energia de ativação para a reação de reticulação de resina epoxídica. Esses autores encontraram valores similares de energia de ativação $\left(\mathrm{E}_{\mathrm{a}}\right)$ pelos métodos de Barrett e pelos mé-

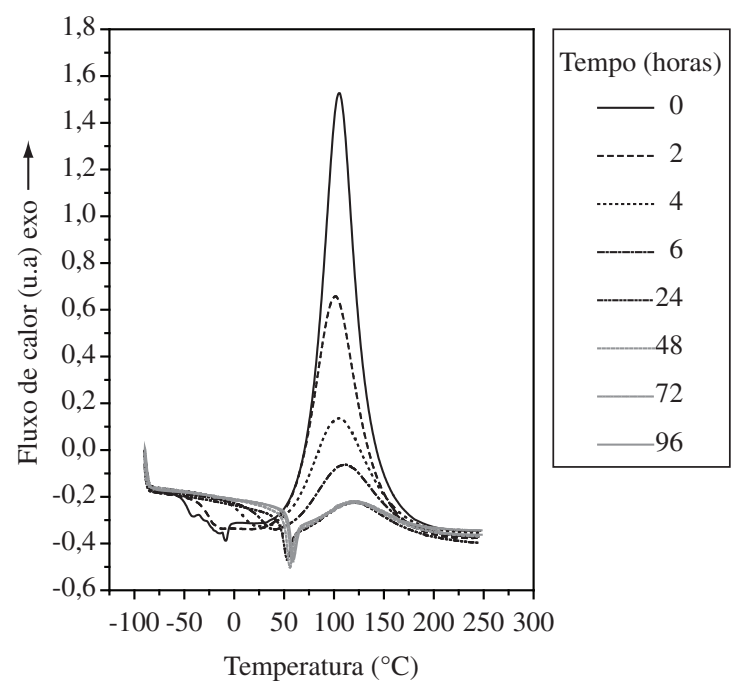

(a)

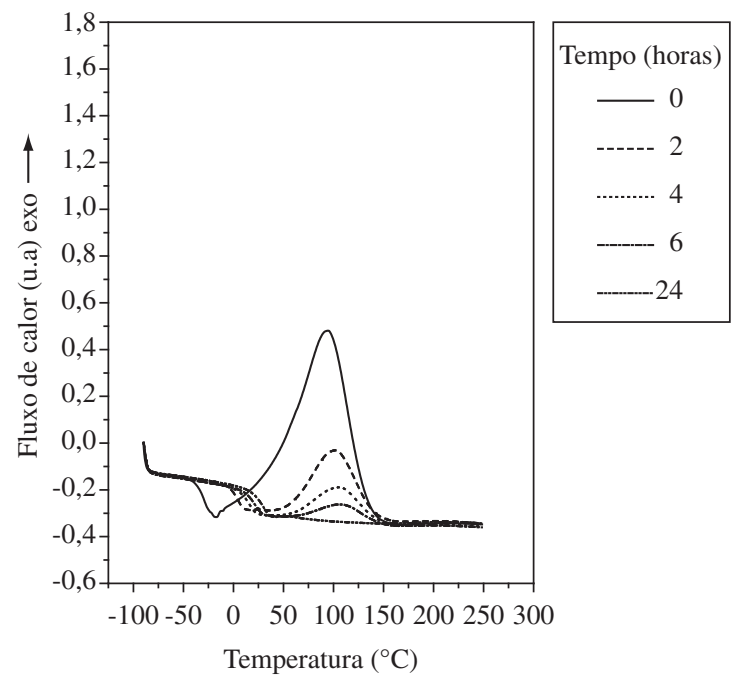

(b)

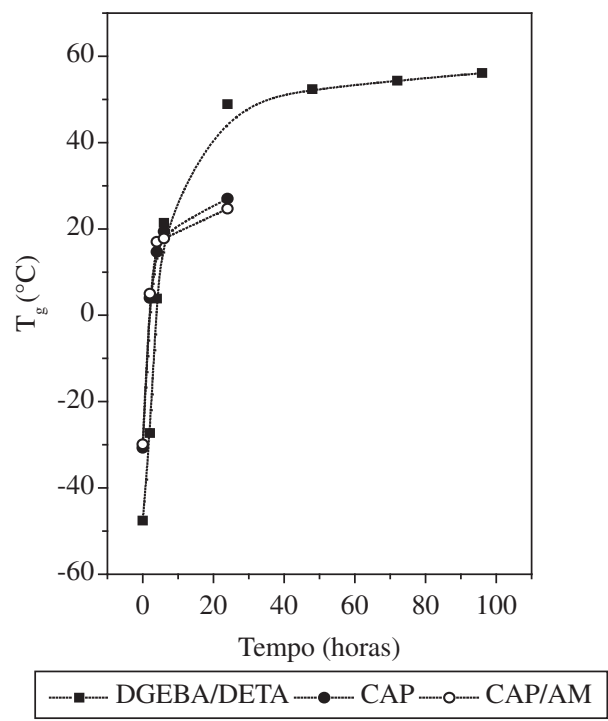

(c)

Figura 4. Curvas DSC obtidas para os adesivos a) DGEBA/DETA; b) CAP; e c) Tg em função do tempo de cura para os adesivos epoxídicos. 
todos que utilizam diferentes taxas de aquecimento como os métodos de Kissinger, Ozawa e meia-altura. A $\mathrm{E}_{\mathrm{a}}$ obtida pelo método de Barrett para toda a extensão da curva de lnk em função do inverso da temperatura para os adesivos em estudo é apresentada na Tabela 3.

$\mathrm{O}$ valor de $\mathrm{E}_{\mathrm{a}}$ é maior para o sistema DGEBA/DETA e diminui quando o CAPCURE é adicionado. A presença do amianto praticamente não altera o valor da $\mathrm{E}_{\mathrm{a}}$ em relação ao adesivo contendo apenas o CAPCURE.

Na Figura 5a são mostradas as curvas de lnk em função do inverso da temperatura para os diferentes adesivos. Notase que estas não são lineares, indicando que não seguem cinética de primeira ordem, no entanto, pelo método de Barrett é possível se obter informação sobre a reação de reticulação, podendo-se identificar, por exemplo, a que conversão ocorre a gelificação pela mudança da inclinação da curva de lnk vs. 1/T ${ }^{[23]}$. A curva obtida para o DGEBA/DETA mostra dois estágios (Figura 5a), sendo que o final do primeiro estágio ocorre em $109{ }^{\circ} \mathrm{C}$ e corresponde a uma conversão de 0,54. Este valor de conversão é próximo ao valor de conversão previsto pela teoria de gelificação de Flory ${ }^{[24]}$, calculado para o ponto de gel em resinas epoxídicas bifuncionais e diaminas tetrafuncionais, estimado em 0,58 de conversão.

A adição do CAPCURE ao adesivo DGEBA/DETA altera o perfil da curva de lnk em função do inverso da temperatura. A mudança de inclinação dessa curva é observada em $57^{\circ} \mathrm{C}$ e em 0,20 de conversão para o adesivo contendo o CAPCURE e $52{ }^{\circ} \mathrm{C}$ e 0,15 para o adesivo CAP/AM. Isto é verificado na prática pela redução do tempo de cura desses adesivos. Estes valores estão bem abaixo do valor previsto por Flory ${ }^{[24]}$ e do valor observado para o sistema DGEBA/DETA, podendo ser atribuídos à presença do CAPCURE, que reagirá com a resina DGEBA, que estará envolvida em duas reações de cura, uma com os grupo mercaptana e outra com o grupo amina, como mostrado na Figura 1.

Os adesivos contendo CAPCURE apresentaram valores de $\mathrm{k}$ superiores aos observados para o DGEBA/DETA em toda a extensão de reação, com velocidade de reação conseqüentemente superior. A adição do amianto ao adesivo CAP causa uma pequena variação nos valores de lnk até a conversão de 0,04 , sendo que acima desse valor as curvas praticamente se sobrepõem.

A energia de ativação também foi calculada pelo método de Borchardt e Daniels para os sistemas em estudo e os valores obtidos para a cura total utilizando-se as curvas DSC ( $\mathrm{t}=0$ horas $)$ são mostrados na Tabela 3. Observa-se uma diferença entre os valores de $\mathrm{E}_{\mathrm{a}}$ obtidos pelos dois métodos, principalmente no

Tabela 3. Energia de ativação $\left(E_{a}\right)$ obtida pelos métodos de Barrett e Borchardt e Daniels (B-D) para os adesivos a base de DGEBA.

\begin{tabular}{ccc}
\hline Adesivo & $\begin{array}{c}\mathbf{E}_{\mathbf{a}}\left(\mathbf{k J} \cdot \mathbf{m o l}^{\mathbf{1}}\right) \\
(\text { Barrett })\end{array}$ & $\begin{array}{c}\mathbf{E}_{\mathbf{a}}\left(\mathbf{k J} \cdot \mathbf{m o l}^{-\mathbf{1}}\right) \\
(\mathbf{B}-\mathbf{D})\end{array}$ \\
\hline DGEBA/DETA & $46 \pm 1$ & $76 \pm 1$ \\
CAP & $31 \pm 1$ & $27 \pm 1$ \\
CAP/AM & $32,8 \pm 0,5$ & $26 \pm 1$ \\
\hline
\end{tabular}

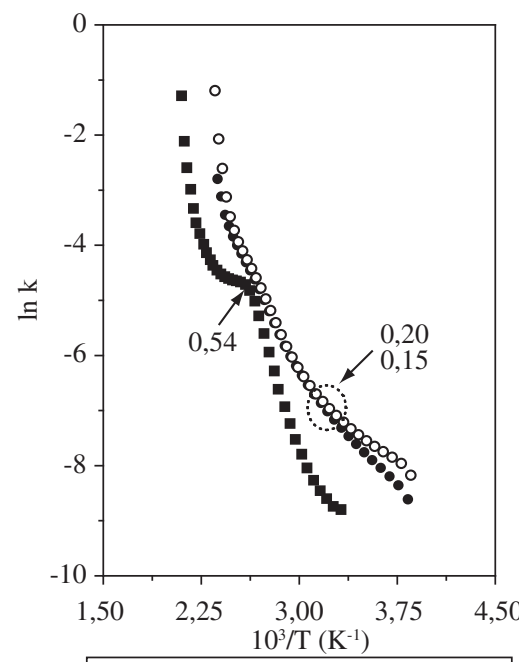

$\bullet$ CAP $\circ \mathrm{CAP} / \mathrm{AM}$-DGEBA/DETA

(a)

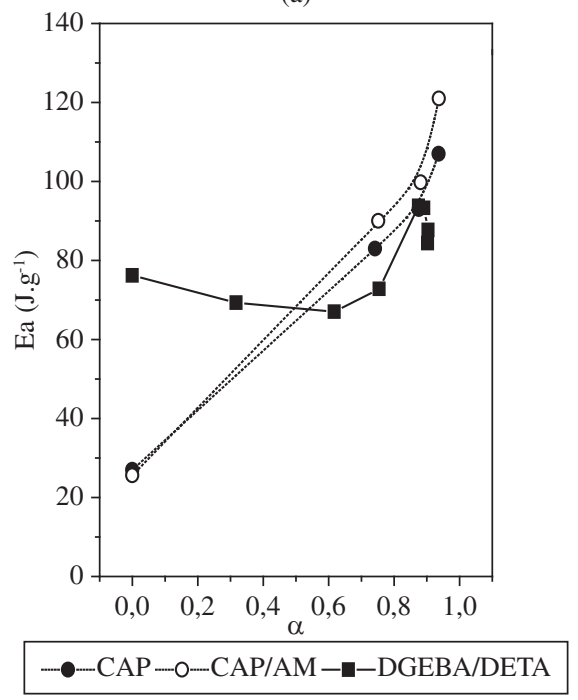

(b)

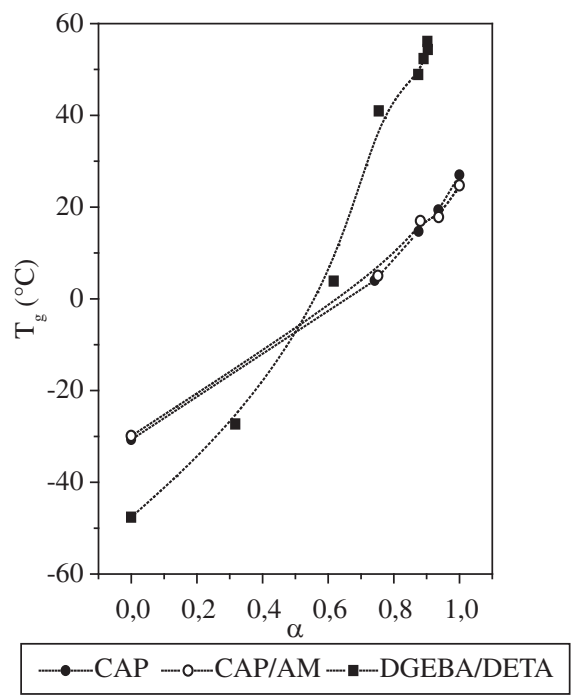

(c)

Figura 5. a) Curvas de lnk vs. 1/T obtidas pelo método de Barrett para os adesivos epoxídicos; b) $\mathrm{E}_{\mathrm{a}}$ em função do grau de conversão ( $\alpha$ ); e c) Tg em função do grau de conversão para os adesivos epoxídicos. 
adesivo DGEBA/DETA o que provavelmente está associado às condições assumidas em cada método. Apesar dessa diferença, observa-se que há coerência entre os valores de $\mathrm{E}_{\mathrm{a}}$, pois independentemente do método que se utilize, os adesivos contendo CAPCURE apresentam valores de $\mathrm{E}_{\mathrm{a}}$ inferiores aos observados para o adesivo DGEBA/DETA, indicando que a reação no sistema contendo CAPCURE é mais rápida.

Para efeito de comparação, utilizou-se o método de Borchardt e Daniels para se calcular a variação da $\mathrm{E}_{\mathrm{a}} \mathrm{em}$ diferentes tempos de cura (Figura $5 b$ ) a partir das curvas DSC mostradas na Figura 4. No caso do DGEBA/DETA, observa-se que a $\mathrm{E}_{\mathrm{a}}$ passa por um mínimo a 0,4 de conversão aumentando até conversão de 0,90 , refletindo o caráter autocatalítico da reação. Neste ponto, observa-se que há uma queda nos valores de energia de ativação, que é coincidente com a estabilização da reação de cura desse adesivo a $25^{\circ} \mathrm{C}$. Os adesivos contendo o CAPCURE inicialmente apresentam valores de $\mathrm{E}_{\mathrm{a}}$ bem inferiores aos observados para o adesivo DGEBA/DETA devido à maior reatividade dos grupos mercaptana, esses valores aumentam conforme a reação avança, provavelmente, devido ao consumo destes grupos. A presença do amianto causa um leve aumento na $\mathrm{E}_{\mathrm{a}}$ em relação ao adesivo sem amianto. Em ambos, a conversão final é próxima de 1 , o que deve estar associada à maior mobilidade das cadeias nos adesivos CAP e CAP/AM que no sistema DGEBA/DETA. Na Figura 5c são apresentadas as curvas da Tg $v s$. $\alpha$ para esses adesivos, confirmando que a $\mathrm{Tg}$ dos adesivos CAP e CAP/AM estão abaixo da temperatura ambiente em toda a faixa de conversão.

\section{Conclusão}

A utilização do CAPCURE no sistema DEGEBA/DETA reduziu o tempo de cura desse adesivo para 24 horas, e possibilitou sua cura completa como observado pelos resultados de FT-MIR/NIR e DSC. Os resultados obtidos pelos tratamentos cinéticos indicaram que a adição do CAPCURE ao sistema DGEBA/DETA torna a reação de cura mais rápida, observando-se maiores valores de $\mathrm{k}$ e menores valores de energia de ativação nos adesivos contendo o CAPCURE.

\section{Referências Bibliográficas}

1. Cranker, K. R. \& Breslau, A. - Ind. Eng. Chem., 48, p.98, (1956).

2. Catálogo Cognis, (2001).

3. Cui, Z.; Lu, C.; Yang, B.; Shen, J.; Su, X. \& Yang, H. Polym., 42, p.1095, (2001).

4. Lowe, G. B. - Int. J. Adhes. Adhes., 17, p.345, (1997).
5. Romão, B. M. V.; Diniz, M. F.; Azevedo, M. F. P.; Lourenço, V. L.; Pardini, L. C. \& Dutra, R. C. L. - Polimeros: Cienc Tecnol, 13, p.173, (2004).

6. Romão, B. M. V.; Diniz, M. F.; Pardini, L. C. \& Dutra, R. C. L. - Polimeros: Cienc Tecnol, 14, p.142, (2004).

7. Romão, B. M. V.; Diniz, M. F.; Azevedo, M. F. P.; Lourenço, V. L.; Pardini, L. C.; Dutra, R. C. L. \& Burel, F. - Polimeros: Cienc Tecnol, 16, p.94, (2006).

8. Cherdoud-Chihani, A.; Mouzali, M. \& Abadie, M. J. M. J. Appl. Polym. Sci. 69, p.1167, (1998).

9. Urbanski, J.; Czerwinski, W.; Janicka, K.; Majewska, F. \& Zowall, H. - "Handbook of analysis of synthetic polymers and plastics", John Wiley, New York (1977).

10. Ooi, S. K.; Cook, W. D.; Simon, G. P. \& Such, C. H. -Polym., 41, p.3639, (2000).

11. Kathryrin, A \& Lee, B. - Appl. Spetrosc. Rev. 28, (3), p.231, (1993).

12. Poisson, N.; Lachenal, G. \& Sautereau, H. - Vib. Spectrosc. 12, p.237, (1996).

13. Weyer, L. G. - Appl. Spectrosc. Rev. 21, p.1, (1985).

14. Núnez, L.; Castro, F. F.; Núnez, M. R. \& Vullanueva, M. J. Appl. Polym. Sci., 77, p.2285, (2000).

15. Grohens, Y.; George, B.; Toyeras, F.; Vebrel, J. \& Laude, B. - Polym. Test., 16, p.417, (1997).

16. ASTM E2041-03: "Standard Method for Estimating Kinetic Parameters by Differential Scanning Calorimeter Using the Borchardt and Daniels Method".

17. Barrett, K. E. J. - J. Appl. Polym. Sci., 11, p.1617, (1967).

18. Wu, L.; Hoa, S. V.; Tan, M. \& That, T. - J. Appl. Polym. Sci., 99, p.580, (2006).

19. Hummel, D. O. \& Scholl, F. - "Atlas of Polymer and Plastics Analysis", Cerlag Chemie GMbH, D 6940 Weinhein, 3, (1981) - Espectros: a) 6424; e b) 5406.

20. Romão, B. V. - "Caracterização e avaliação de sistemas epoxídicos utilizados na indústria aeroespacial”, Dissertação de Mestrado, Instituto Tecnológico da Aeronáutico, Brasil, (2004).

21. Goddu, R. F. - Adv. Anal. Chem. Instrum., 1, p.347, (1960).

22. R. B. - Prime, in: "Thermal Characterization of Polymeric Materials", Edith A. Turi (ed.); Academic Press, p.435 New York (1981).

23. Miranda, M. I. G.; Tomedi, C.; Bica, C. I. D. \& Samios, D. - Polym., 38, p. 1017, (1997).

24. Flory, P. J. - "Principles of Polymer Chemistry", Cornell Univerty Press, London, (1953).

Enviado: $26 / 12 / 07$

Reenviado: 23/06/08

Aceito: $11 / 07 / 08$ 